\title{
CORRESPONDENCE
}

To the Editor: Based on a valid comment received from Professor Rudi Schmid, we wish to submit an amendment to our paper on bilirubin toxicity, published in your July 2003 issue (1). Rudi has advised us that Equation 1, which we utilized to calculate the unbound concentrations (BF) of unconjugated bilirubin (UCB), is inaccurate at $\mathrm{BF}$ values above aqueous saturation $(70 \mathrm{nM})$. The now octogenarian guru of the bilirubin field was very perceptive to detect this oversight on our part.

Equation 1, derived from Brodersen's Eq. 15 in (2), is based on a model in which unbound bilirubin (UCB) monomers are in equilibrium with monomers bound at two independent sites on albumin. This is valid when the unbound UCB concentration, $\mathrm{BF}$, is below aqueous saturation $(70 \mathrm{nM})$, but at $\mathrm{BF}$ values above saturation, errors arise due to self-association of unbound UCB monomers (3). Thus, as BF increases above saturation, oligomers and larger aggregates of UCB will constitute a progressively larger proportion of the total unbound $\mathrm{UCB}$, and the monomer concentrations (BF values calculated from Eq. 1) will progressively underestimate the true total unbound UCB concentrations.

Among the studies evaluated in our meta-analysis (Tables 1 and 2 in the original paper), the error is relatively small at calculated BF values of 71 and $85 \mathrm{nM}(4-6)$, which are only slightly above aqueous saturation. In addition, in the studies done without albumin , the unbound UCB concentrations are equal to the measured total UCB concentrations of $250 \mathrm{nM}$ and $400-500$ $\mathrm{nM}$ (7-10), without using Eq. 1 and without assumptions about states of aggregation. No toxicity was observed in any of the studies at BF values below $70 \mathrm{nM}$. Therefore, despite the inaccuracy of our calculated higher $\mathrm{BF}$ values, our conclusion is still valid that "UCB can impair various cellular functions of astrocytes and neurons exposed to BF near or modestly above its aqueous solubility limit, at which UCB exists as soluble oligomers and metastable microaggregates."
Jay Donald Ostrow

Research Service (151L),

VA Puget Sound Health Care System - Seattle Division, and GI/Hepatology Division, Univ. Washington School of Medicine

Seattle, WA 98108-1597, U.S.A. Jdostrow@medicine.washington.edu

Lorella Pascolo

Claudio Tiribelli

Centro Studio Fegato

AREA Science Park and

Department BBCM, University of Trieste

34012 Trieste, Italy

\section{Reference List}

1. Ostrow JD, Pascolo L, Tiribelli C 2003 Reassessment of the unbound concentrations of unconjugated bilirubin in relation to neurotoxicity in vitro. Pediatr Res 54:98-104

2. Brodersen R 1986 Aqueous solubility, albumin binding and tissue distribution of bilirubin. In: Ostrow JD (ed) Bile Pigments and Jaundice; Molecular, Metabolic and Medical Aspects. Marcel Dekker, New York, pp 157-181

3. Mukerjee P, Ostrow JD, Tiribelli C 2002 Low solubility of unconjugated bilirubin in dimethylsulfoxide - water systems: implications for $\mathrm{pK}_{\mathrm{a}}$ determinations. BMC Biochemistry $3: 17$

4. Chuniaud L, Dessante M, Chantoux F, Blondeau JP, Francon J, Trivin F 1996 Cytotoxicity of bilirubin for human fibroblasts and rat astrocytes in culture. Effect of the ratio of bilirubin to serum albumin. Clin Chim Acta 256:103-114

5. Silva RFM, Rodrigues CMP, Brites D 2001 Bilirubin-induced apoptosis in cultured rat neural cells is aggravated by chenodeoxycholic acid but prevented by ursodeoxycholic acid. J Hepatology 34:402-408

6. Rodrigues CMP, Sola S, Brites D 2002 Bilirubin induces apoptosis via the mitochondrial pathway in developing rat brain neurons. Hepatology 35:1186-1195

7. Amit Y, Brenner T 1993 Age-dependent sensitivity of cultured rat glial cells to bilirubin toxicity. Exp Neurol 121:248-255

8. Dore S, Snyder SH 1999 Neuroprotective action of bilirubin against oxidative stress in primary hippocampal cultures. Ann NY Acad Sci 890:167-172

9. Grojean S, Koziel V, Vert P, Daval JL 2000 Bilirubin induces apoptosis via activation of NMDA receptors in developing rat brain neurons. Exp Neurol 166:334-341

10. Grojean S, Lievre V, Koziel V, Vert P, Daval JL 2001 Bilirubin exerts additional toxic effects in hypoxic cultured neurons from the developing rat brain by the recruitment of glutamate neurotoxicity. Pediatr Res 49:507-513 